\title{
REGULARITY OF SPHERICAL MEANS AND LOCALIZATION OF SPHERICAL HARMONIC EXPANSIONS
}

\author{
LEONARDO COLZANI
}

(Received 10 October 1984; revised 11 March 1985)

Communicated by G. Brown

\begin{abstract}
Let $G / K$ be a compact symmetric space, and let $G=K A K$ be a Cartan decomposition of $G$. For $f$ in $L^{1}(G)$ we define the spherical means $f(g, t)=\int_{K} \int_{K} f\left(g k t k^{\prime}\right) d k d k^{\prime}, g \in G, t \in A$. We prove that if $f$ is in $L^{p}(G), 1 \leqslant p \leqslant 2$, then for almost every $g \in G$ the functions $t \mapsto f(g, t)$ belong to certain Soblev spaces on $A$. From these regularity results for the spherical means we deduce, if $G / K$ is a compact rank one symmetric space, a theorem on the almost everywhere localization of spherical harmonic expansions of functions in $L^{2}(G / K)$.
\end{abstract}

1980 Mathematics subject classification (Amer. Math. Soc.): 42 C 10, 43 A 15

Keywords and phrases: symmetric spaces, spherical means, localization.

\section{Sunto}

Sia $G / K$ uno spazio simmetrico compatto, e sia $G=K A K$ una decomposizione di Cartan del gruppo $G$. Se $f \in L^{i}(G)$, si definiscono le medie sferiche $f(g, t)=\int_{K} \int_{K} f\left(g k t k^{\prime}\right) d k d k^{\prime}, g \in G, t \in A$. Si dimostra che se $f \in L^{p}(G), 1 \leqslant p \leqslant 2$, allora per quasi ogni $g \in G$, le funzioni $t \rightarrow f(g, t)$ appartengono a certi spazi di Sobolev su $A$. Da questi risultati di regolarita si deducono dei risultati di localizzazione quasi ovunque per sviluppi in serie di armoniche sferiche di funzioni in $L^{2}(G / K)$, quando $G / K$ è uno spazio simmetrico compatto di rango uno.

\section{Introduction}

For $f \in L_{\mathrm{loc}}^{1}\left(R^{N}\right), x \in R^{n}$, and $t \geqslant 0$, we define the spherical means $f(x, t)$ by

$$
f(x, t)=\int_{S^{N-1}} f(x-t y) d \sigma(y),
$$

(c) 1986 Australian Mathematical Society $0263-6115 / 86 \$ A 2.00+0.00$ 
where $\sigma$ is the surface measure of the unit sphere $S^{N-1}$ in $R^{N}$. E. M. Stein proved in [16] that if $f$ is in $L^{p}\left(R^{N}\right)$, with $N \geqslant 3$ and $p>N /(N-1)$, then for almost every $x \in R^{N}$ the function $t \rightarrow f(x, t)$ is bounded and continuous in $t \in[0,+\infty)$ and later J. Peyrière and P. Sjölin in [14] and D. M. Oberlin and E. M. Stein in [13] proved

Theorem A. Let $N \geqslant 3$, let $p>N /(N-1)$, and let $f \in L P_{o c}\left(R^{N}\right)$. Then for almost every $x \in R^{n}, f(x, \cdot)$ belongs to the Lipschitz space $\Lambda_{N(1-1 / p)-1}^{\mathrm{loc}}((0,+\infty))$.

Part of this result is also implicitly contained in [7]. A more precise result, due again to P. Sjölin [15], is the following theorem. Denote by $L_{\alpha}^{2}(R), \alpha \geqslant 0$, the Sobolev space

$$
L_{\alpha}^{2}(R)=\left\{h \in L^{2}(R):\|h\|_{L_{\alpha}^{2}}=\left(\int_{-\infty}^{+\infty}(1+|s|)^{2 \alpha}|\hat{h}(s)|^{2} d s\right)^{1 / 2}<+\infty\right\} .
$$

Theorem B. Let $\phi \in C^{\infty}(R)$ be such that Supp $\phi \subset(0,+\infty)$. If $N \geqslant 2$, if $N /(N-1 / 2) \leqslant p \leqslant 2$, if $\alpha=N(1-1 / p)-1 / 2$, and if $f \in L^{p}\left(R^{N}\right)$, then

$$
\left(\int_{R^{N}}\|\phi(\cdot) f(x, \cdot)\|_{L_{\alpha}^{2}}^{2} d x\right)^{1 / 2} \leqslant c\|f\|_{p}
$$

Since $L_{\alpha+1 / 2}^{2}(R)$ is contained in $\Lambda_{\alpha}(R)$, Theorem B implies Theorem A.

In this paper we shall extend these results from $R^{N}$ to any compact symmetric space. Our approach to the study of spherical means in these spaces is essentially group-theoretic, and it is based on the theory of spherical functions. We have found this point of view illuminating: in this general setting proofs are simple, and the results quite general.

In Section 1 we shall prove an analog of Theorem B for any compact symmetric space, but special emphasis will be put on the rank one case. In Section 2 we shall apply the results obtained in Section 1 to the study of almost everywhere localization of spherical harmonic expansions of $L^{2}$-functions on compact rank one symmetric spaces.

We are happy to thank Christopher Meaney for several helpful discussions concerning the subject of this paper.

\section{Regularity of spherical means}

Let $(G, K)$ be a compact symmetric pair, and let $X=G / K$ be the associated compact symmetric space (see [4] and [10] for the definitions and main properties). 
We shall always identify functions and distributions on $X$ with right $K$-invariant functions and distributions on $G$. Let $G=K A K$ be a Cartan decomposition of $G$. Then, if $g=k t k^{\prime}$, with $k$ and $k^{\prime}$ in $K$ and $t$ in $A$, the Haar measure $d g$ of $G$ can be decomposed as $d g=d k w(t) d t d k^{\prime}$, for an appropriate weight $w$ on $A$. If $f$ is in $L^{1}(G)$ we define the spherical means $f(g, t), g \in G, t \in A$, by

$$
f(g, t)=\int_{K} \int_{K} f\left(g k t k^{\prime}\right) d k d k^{\prime} .
$$

An application of Fubini's theorem shows that for every $g \in G, f(g, t)$ is well defined for almost every $t \in A$. Moreover it is immediate to verify that $f(g, t)$, as function of $g$, is constant on the left cosets of $K$ in $G$; hence it is properly a function on $X$.

Let $\hat{G}_{k}$ be the subset of the dual of $G$ consisting of equivalence classes of class one representations, and let $\left\{\phi_{\lambda}\right\}_{\lambda \in \hat{G}_{K}}$ be the associated system of zonal spherical functions. If $f \in L^{2}(G)$ is right $K$-invariant, the Peter-Weyl theorem becomes

$$
f=\sum_{\lambda \in \hat{G}_{K}} f * d_{\lambda} \phi_{\lambda}
$$

where $d_{\lambda}$ denotes the dimension of $\lambda$, and where $*$ denotes the convolution. And since every zonal spherical function $\phi_{\lambda}$ satisfies the equation

$$
\int_{K} \phi_{\lambda}\left(g k g^{\prime}\right) d k=\phi_{\lambda}(g) \phi_{\lambda}\left(g^{\prime}\right),
$$

the spherical means $f(g, t)$ have the expansion

$$
f(g, t)=\sum_{\lambda \in \hat{G}_{K}}\left(f * d_{\lambda} \phi_{\lambda}(g)\right) \phi_{\lambda}(t) .
$$

These spherical means are then nice convolution operators that can be defined not only for functions in $L^{1}(G)$, but also for distributions on $G$. Also the above expansion suggests that it is natural to study the regularity of $f(g, t)$ in terms of some sort of Sobolev space associated with the system $\left\{\phi_{\lambda}\right\}_{\lambda \in \hat{\sigma}_{k}}$. If $t \in A$, we set $\psi_{\lambda}(t)=d_{\lambda}^{1 / 2} \phi_{\lambda}(t)$. Then the system $\left\{\psi_{\lambda}\right\}_{\lambda \in \hat{G}_{K}}$ is orthonormal in $L^{2}(A, w(t) d t)$. We define the Sobolev space $L_{\alpha}^{2}(A, w(t) d t),-\infty<\alpha<+\infty$, to be the set of all distributions $h=\Sigma_{\lambda \in \hat{G}_{k}} \hat{h}(\lambda) \psi_{\lambda}$, with

$$
\|h\|_{L_{\alpha}^{2}}=\left(\sum_{\lambda \in \hat{G}_{k}}(1+|\lambda|)^{2 \alpha}|\hat{h}(\lambda)|^{2}\right)^{1 / 2}<+\infty .
$$

Here $|\lambda|$ denotes the norm of the highest weight corresponding to $\lambda$, and $\hat{h}(\lambda)$ denotes the $\lambda$ th Fourier coefficient of $h$, i.e., if $h$ is in $L^{1}(A, w(t) d t)$, then

$$
\hat{h}(\lambda)=\int_{A} h(t) \overline{\psi_{\lambda}(t)} w(t) d t .
$$


We are now ready to state our theorem. We begin by stating the results for compact rank one symmetric spaces. The situation for higher ranks is a bit more complicated, and in this case we shall only sketch the proofs.

TheOREM 1.1. Let $X=G / K$ be a compact rank one symmetric space of real dimension $N$.

(i) If the distribution $f$ belongs to the Hardy space $H^{p}(X), 0<p \leqslant 1$, and if $\alpha=N(1-1 / p)-1 / 2$, then

$$
\left(\int_{G}\|f(g, \cdot)\|_{L_{\alpha}^{2}}^{2} d g\right)^{1 / 2} \leqslant c\|f\|_{H^{p}}
$$

(ii) If $f$ is in $L^{1}(X)$, and if $\alpha<-1 / 2$, then

$$
\left(\int_{G}\|f(g, \cdot)\|_{L_{\alpha}^{2}}^{2} d g\right)^{1 / 2} \leqslant c\|f\|_{1} .
$$

(iii) If $f$ is in $L^{p}(X), 1<p \leqslant 2$, and if $\alpha=N(1-1 / p)-1 / 2$, then

$$
\left(\int_{G}\|f(g, \cdot)\|_{L_{\alpha}^{2}}^{2} d g\right)^{1 / 2} \leqslant c\|f\|_{p} .
$$

These results are essentially the best possible.

The Hardy spaces $H^{p}(X), 0<p<+\infty$, are studied in some detail in [6]. However, for the case $p \geqslant 1$, and for the purpose of this paper, it is enough to know [5]. Before going into the (easy) proof of the theorem, we need to introduce some notation (see [1], [4], [9], [10], [17]).

If $X=G / K$ is a compact rank one symmetric space of dimension $N$, and if $G=K A K$ is a Cartan decomposition of $G$, then, after a suitable normalization, we can identify $A$ with the torus $T=[-\pi, \pi)$, and

$$
d g=d k w(t) d t d k^{\prime}=d k|\sin t / 2|^{2 a+1}|\cos t / 2|^{2 b+1} d t d k^{\prime},
$$

where $a=N / 2-1$, and where $b \leqslant a$ depends on the particular $X . \hat{G}_{K}$ is naturally indexed by the nonnegative integers (and by the even nonnegative integers if $X$ is a real projective space), and the zonal spherical functions are closely related to the Jacobi polynomials $\left\{P_{n}^{(a, b)}\right\}_{n=0}^{+\infty}$ : if $g=k t k^{\prime}$, with $k$, $k^{\prime} \in K$, and with $t \in A(=[-\pi, \pi))$, then

$$
\phi_{n}(g)=\frac{P_{n}^{(a, b)}(\cos t)}{P_{n}^{(a, b)}(1)} .
$$

The dimension $d_{n}$ of the $n$th representation can be computed explicitly in terms of $a, b$, and $n$. However, it is enough to say here that there exist two positive 
constants $c_{1}$ and $c_{2}$ such that, for every $n$,

$$
c_{1}(1+n)^{N-1} \leqslant d_{n} \leqslant c_{2}(1+n)^{N-1} .
$$

Proof OF The TheOREM. Observe that, due to the $K$-bi-invariance of $\phi_{n}$, if $f$ is an integrable function on $G$, then we have

$$
\begin{aligned}
f * d_{n} \phi_{n}(g) & =\int_{G} f\left(g s^{-1}\right) d_{n} \phi_{n}(s) d s \\
& =\int_{K} \int_{A} \int_{K} f\left(g k t k^{\prime}\right) d_{n} \phi_{n}\left(t^{-1}\right) d k w(t) d t d k^{\prime} \\
& =\int_{A} f(g, t) d_{n} \overline{\phi_{n}(t)} w(t) d t \\
& =d_{n}^{1 / 2}(f(g, \cdot))(n) .
\end{aligned}
$$

Thus

$$
\begin{aligned}
\int_{G}\|f(g, \cdot)\|_{L_{\alpha}^{2}}^{2} d g & =\int_{G} \sum_{n=0}^{+\infty}(1+n)^{2 \alpha}|(f(g, \cdot))(n)|^{2} d g \\
& =\sum_{n=0}^{+\infty}(1+n)^{2 \alpha} d_{n}^{-1} \int_{G}\left|f * d_{n} \phi_{n}(g)\right|^{2} d g \\
& =\sum_{n=0}^{+\infty}(1+n)^{2 \alpha} d_{n}^{-1}\left\|f * d_{n} \phi_{n}\right\|_{2}^{2} .
\end{aligned}
$$

We first prove (ii). If $f$ is in $L^{1}(X)$, then we have

$$
\left\|f * d_{n} \phi_{n}\right\|_{2} \leqslant\|f\|_{1}\left\|d_{n} \phi_{n}\right\|_{2}=d_{n}^{1 / 2}\|f\|_{1} \text {. }
$$

Hence

$$
\int_{G}\|f(g, \cdot)\|_{L_{\alpha}^{2}}^{2} d g \leqslant \sum_{n=0}^{+\infty}(1+n)^{2 \alpha}\|f\|_{1}^{2},
$$

and this series converges if $\alpha<-1 / 2$. This proves (ii).

To prove (i) and (iii) we use the fact that $d_{n} \sim(1+n)^{N-1}$. Thus,

$$
\int_{G}\|f(g, \cdot)\|_{L_{\alpha}^{2}}^{2} d g-\sum_{n=0}^{+\infty}(1+n)^{2 \alpha-N+1}\left\|f * d_{n} \phi_{n}\right\|_{2}^{2} .
$$

If $f$ is in $L^{2}(X)$ and $\alpha=(N-1) / 2$, the desired result is a consequence of the Plancherel formula (and the theorem is sharp in this case). To treat the case $p<2$, we introduce the "fractional integral operators" $\left\{I^{\beta}\right\}_{\beta \in R}$, defined by

$$
I^{\beta} f=\sum_{n=0}^{+\infty}(1+n)^{-\beta} f * d_{n} \phi_{n} \text {. }
$$


It is proved in [6] that if $0<p \leqslant q<+\infty$, and if $\beta=N(1 / p-1 / q)$, then the operator $I^{\beta}$ maps $H^{p}(X)$ into $H^{q}(X)$ continuously (here of course $H^{p}(X)=$ $L^{p}(X)$ if $\left.1<p<+\infty\right)$. In particular, if $p \leqslant 2$, then $I^{N(1 / p-1 / 2)}$ maps $H^{p}(X)$ into $L^{2}(X)$ continuously. This statement is equivalent to the following inequality:

$$
\left(\sum_{n=0}^{+\infty}(1+n)^{-2 N(1 / p-1 / 2)}\left\|f * d_{n} \phi_{n}\right\|_{2}^{2}\right)^{1 / 2} \leqslant c\|f\|_{H^{p}},
$$

and (i) and (iii) follow.

To prove that the results in (ii) and (iii) are sharp, let us consider the "Poisson kernel"

$$
P_{r}(g)=\sum_{n=0}^{+\infty} r^{n} d_{n} \phi_{n}(g), \quad 0 \leqslant r<1 .
$$

Since for every $g$ in $G, P_{r}(g)>0$ (see [1] and [17]), we have $\left\|P_{r}\right\|_{1}=1$. Also

$$
\left\|P_{r}\right\|_{2}=\left(\sum_{n=0}^{+\infty} r^{2 n} d_{n}\right)^{1 / 2} \sim\left(\sum_{n=0}^{+\infty} r^{2 n}(1+n)^{N-1}\right)^{1 / 2} \sim(1-r)^{-N / 2}
$$

and

$$
\left\|P_{r}\right\|_{\infty}=\sum_{n=0}^{+\infty} r^{n} d_{n} \sim \sum_{n=0}^{+\infty} r^{n}(1+n)^{N-1} \sim(1-r)^{-N},
$$

and, by interpolation,

$$
\left\|P_{r}\right\|_{p} \sim(1-r)^{N(1 / p-1)} \text { if } 1 \leqslant p \leqslant+\infty .
$$

(If we had better estimates for $\left\|P_{r}\right\|_{p}, p \neq 1,2,+\infty$, then a new interpolation between $p$ and 1 , or $p$ and $+\infty$, would provide better estimates also for $\left\|P_{r}\right\|_{2}$, but this would be a contradiction.) Hence

$$
\begin{array}{rlrl}
\left(\int_{G}\left\|P_{r}(g, \cdot)\right\|_{L_{\alpha}^{2}}^{2} d g\right)^{1 / 2} & =\left(\sum_{n=0}^{+\infty}(1+n)^{2 \alpha} r^{2 n}\right)^{1 / 2} & \\
& \sim 1 & \text { if } \alpha<-1 / 2 \\
& -(1-\log (1-r))^{1 / 2} & \text { if } \alpha=-1 / 2, \\
& \sim(1-r)^{-1 / 2-\alpha} & & \text { if } \alpha>-1 / 2 .
\end{array}
$$

Letting $r \rightarrow 1^{-}$, we see that

$$
\left(\int_{G}\left\|P_{r}(g, \cdot)\right\|_{L_{\alpha}^{2} d g}^{2}\right)^{1 / 2} \leqslant c\left\|P_{r}\right\|_{p}
$$

only if $\alpha<-1 / 2$ and $p=1$, or if $\alpha \leqslant N(1-1 / p)-1 / 2$ and $1<p \leqslant 2$. 
To prove that the results in (i) are sharp, we can consider instead of the Poisson kernel $P_{r}$ its "radial derivatives" $\left(d^{k} / d r^{k}\right) P_{r}$. (It can be proved that if $k>$ $N(1 / p-1)$,

$$
\left.\left\|\frac{d^{k}}{d r^{k}} P_{r}\right\|_{H^{p}} \sim(1-r)^{N(1 / p-1)-k} .\right)
$$

The proof of the theorem is now complete.

REMARK 1.2. It is possible to prove part (iii) and part (i) of Theorem 1.1 with $p=1$, without using (explicitly) the fractional integral operators $\left\{I^{\beta}\right\}_{\beta \in R}$. Since this approach is somewhat more elementary, it will be sketched here. R. R. Coifman and G. Weiss proved in [5] that if the function $f$ is in the atomic Hardy space $H^{1}\left(S^{N}\right)$, then the following Hardy inequality holds:

$$
\sum_{n=0}^{+\infty}(1+n)^{-(N+1) / 2}\left\|f * d_{n} \phi_{n}\right\|_{2} \leqslant c\|f\|_{H^{1}} .
$$

This result has been subsequently extended to any compact symmetric space by F. Cazzaniga and E. Giacalone in [3]. Actually the same techniques used by these authors yield the inequality

$$
\left(\sum_{n=0}^{+\infty}(1+n)^{-N}\left\|f * d_{n} \phi_{n}\right\|_{2}^{2}\right)^{1 / 2} \leqslant c\|f\|_{H^{1}},
$$

which is valid for every compact rank one symmetric space of dimension $N$. Interpolation between $H^{1}(X)$ and $L^{2}(X)$ then gives

$$
\left(\sum_{n=0}^{+\infty}(1+n)^{-2 N(1 / p-1 / 2)}\left\|f * d_{n} \phi_{n}\right\|_{2}^{2}\right)^{1 / 2} \leqslant c\|f\|_{p}
$$

if $1<p \leqslant 2$. This is just the result in fractional integration which we used in the proof of part (iii) of the theorem.

REMARK 1.3. Theorem 1.1 is the analog for compact rank one symmetric spaces of Theorem B for $R^{N}$. Indeed it is not difficult to state and prove also an analog of Theorem A: let $N \geqslant 3$, let $p>N /(N-1)$, and let $\delta>0$; if $f$ is in $L^{p}(X)$, then for almost every $g \in G$, the spherical means $f(g, \cdot)$ belong to the Lipschitz space $\Lambda_{N(1-1 / p)-1}([\delta, \pi-\delta])$. Indeed, it follows from the asymptotic formula of the Jacobi polynomials [17] that, away from the "antipodal points" 0 and $\pi$, the functions $\psi_{n}(t)$ behave like the trigonometric polynomials $\cos (n t)$, and the Sobolev spaces $L_{\alpha+1 / 2}^{2}(A, w(t) d t), \alpha>0$, are imbedded into the Lipschitz spaces $\Lambda_{\alpha}([\delta, \pi-\delta])$. 
Let us now consider the analog of Theorem 1.1 for compact symmetric spaces of rank greater than one.

THEOREM 1.4. Let $X=G / K$ be a compact symmetric space of dimension $N$ and rank $Q$, and let $M$ be a constant such that for every $\lambda \in \hat{G}_{K}$, we have $d_{\lambda} \geqslant$ $c(1+|\lambda|)^{M}$.

(i) If $f$ is in $L^{1}(X)$, and if $\alpha<-Q / 2$, then

$$
\left(\int_{G}\|f(g, \cdot)\|_{L_{\alpha}^{2}}^{2} d g\right)^{1 / 2} \leqslant c\|f\|_{1} .
$$

(ii) If $f$ is in $L^{p}(X), 1<p<2$, and if $\alpha<(M+Q)(1-1 / p)-Q / 2$, then

$$
\left(\int_{G}\|f(g, \cdot)\|_{L_{\alpha}^{2}}^{2} d g\right)^{1 / 2} \leqslant c\|f\|_{p} .
$$

(iii) If $f$ is in $L^{2}(X)$, and if $\alpha=M / 2$, then

$$
\left(\int_{G}\|f(g, \cdot)\|_{L_{\alpha}^{2} d g}^{2}\right)^{1 / 2} \leqslant c\|f\|_{2} .
$$

The best choice for the constant $M$ is determined, through the Weyl dimension formula, by the Lie algebras of $G$ and $K$. In any case $M \leqslant N-Q$ (see [8]).

Proof OF THE THEOREM. (i) and (iii) are proved as in Theorem 1.1, and (ii) follows by interpolation.

It is not difficult to show that the estimates (i) and (iii) are sharp, but it is likely that the estimates (ii) can be slightly ameliorated.

\section{Localization of spherical harmonic expansions}

It is well known that the classical Riemann localization theorem for Fourier series on the one dimensional torus does not extend to an arbitrary Lie group or symmetric space. In particular C. Meaney has proved in [11] that if $X$ is a compact rank one symmetric space of dimension $N$ and diameter $\pi$, and if $x_{0} \in X$, then there exists $f \in L^{2 N /(N+1)}(X)$, such that $f$ is zonal about $x_{0}$, $f(x)=0$ on the ball of center $x_{0}$ and radius $\pi / 2$, and $\sum_{n=0}^{+\infty} f * d_{n} \phi_{n}(x)$ diverges for almost every $x$ in $X$.

Despite this, the following positive result is known. 
THEOREM 2.1. Let $X$ be a compact rank one symmetric space. If $f \in L^{2}(X)$ is zero almost everywhere on an open set $U \subseteq X$, then $\sum_{n=0}^{+\infty} f * d_{n} \phi_{n}(x)$ converges to zero for almost every $x$ in $U$.

This result has been proved independently by A. I. Bastis and C. Meaney in [2] and [12]. We shall here give another proof of this theorem which is based on our previous results on the regularity of the spherical means, and with the hope that the techniques used will be useful in other contexts.

Proof of THE THeOREM. Let $D_{n}=\sum_{j=0}^{n} d_{j} \phi_{j}$ be the " $n$th Dirichlet kernel" of $X$. By the Christoffel-Darboux formula, if $g=k t k^{\prime}$, we have

$$
\begin{aligned}
D_{n}(g) & =c_{0}(n) \frac{P_{n+1}^{(a, b)}(1) P_{n}^{(a, b)}(\cos t)-P_{n}^{(a, b)}(1) P_{n+1}^{(a, b)}(\cos t)}{1-\cos t} \\
& =c(n) \frac{\phi_{n}(t)-\phi_{n+1}(t)}{1-\cos t},
\end{aligned}
$$

with $c_{0}(n)$ and $c(n)$ positive constants, and $c(n) \sim(1+n)^{N-1}$. (This follows from [17].) Let $f$ be an integrable function. Then

$$
\begin{aligned}
\sum_{j=0}^{n} f * d_{j} \phi_{j}(g) & =\int_{G} f\left(g s^{-1}\right) D_{n}(s) d s \\
& =c(n) \int_{A} f(g, t) \frac{\phi_{n}(t)-\phi_{n+1}(t)}{1-\cos t} w(t) d t .
\end{aligned}
$$

Suppose now that $f$ vanishes in a neighborhood of the point $g$; then also $f(g, t)$ as a function of $t$, vanishes in a neighborhood of $t=0$. And so, if $f(g, \cdot)$ is in $L_{\alpha}^{2}(A, w(t) d t)$, also $f(g, \cdot) /(1-\cos (\cdot))$ is in $L_{\alpha}^{2}(A, w(t) d t)$ (see the remark at the end of the proof). In particular, if $n \rightarrow+\infty$, then

$$
\begin{aligned}
\int_{A} \frac{f(g, t)}{1-\cos t} \phi_{n}(t) w(t) d t & =d_{n}^{1 / 2}\left(\frac{f(g, \cdot)}{1-\cos (\cdot)}\right) \hat{(n)} \\
& =o\left((1+n)^{-(N-1) / 2-\alpha}\right)
\end{aligned}
$$

and similarly

$$
\int_{A} \frac{f(g, t)}{1-\cos t} \phi_{n+1}(t) w(t) d t=o\left((1+n)^{-(N-1) / 2-\alpha}\right) .
$$

Collecting these estimates, if $\alpha=(N-1) / 2$, and if $n \rightarrow+\infty$, we obtain

$$
\sum_{j=0}^{n} f * d_{j} \phi_{j}(g)=o(1)
$$

and the theorem follows from the fact that if $f$ is in $L^{2}(X)$, then for almost every $g$ in $G, f(g, \cdot)$ is in $L_{(N-1) / 2}^{2}(A, w(t) d t)$. 
REMARK 2.2. In the proof of the theorem we used the fact that the product $v \cdot h$ of a function $h$ in the Sobolev space $L_{\alpha}^{2}(A, w(t) d t)$ and a function $v$ in $C^{\infty}(A)=\bigcap_{\beta \in R} L_{\beta}^{2}(A, w(t) d t)$, is still in $L_{\alpha}^{2}(A, w(t) d t)$. This must be a well known fact, and also one can readily believe it. In any case, here is a sketch of a proof. Since $\|v \cdot h\|_{L_{\alpha}^{2}} \leqslant \sum_{n=0}^{+\infty}|\hat{v}(n)|\left\|\psi_{n} h\right\|_{L_{\alpha}^{2}}$, and since for every $\beta \in R, \hat{v}(n)=$ $o\left(n^{-\beta}\right)$ as $n \rightarrow+\infty$, it is enough to show that

$$
\left\|\psi_{n} h\right\|_{L_{\alpha}^{2}} \leqslant c(1+n)^{\gamma}\|h\|_{L_{\alpha}^{2}}
$$

for some constants $c$ and $\gamma$.

It is shown in [1] that the product $\psi_{n} \psi_{j}$ admits the linearization

$$
\psi_{n}(t) \psi_{j}(t)=\sum_{m=|n-j|}^{n+j} D(j, m, n) \psi_{m}(t),
$$

for some nonnegative constants $\{D(j, m, n)\}_{m=|n-j|}^{n+j}$. Hence

$$
\begin{aligned}
\sum_{m=|n-j|}^{n+j} D(j, m, n) & \leqslant \frac{\psi_{n}(0) \psi_{j}(0)}{\min _{|n-j| \leqslant m \leqslant n+j} \psi_{m}(0)} \\
& \leqslant c \min \left((1+n)^{N-1},(1+j)^{N-1}\right) .
\end{aligned}
$$

Using these estimates, and the Hardy-Littlewood maximal theorem, we obtain

$$
\begin{aligned}
& \left\|\psi_{n} h\right\|_{L_{\alpha}^{2}}=\left(\sum_{j=0}^{+\infty}(1+j)^{2 \alpha}\left|\sum_{m=|n-j|}^{n+j} D(j, m, n) \hat{h}(m)\right|^{2}\right)^{1 / 2} \\
& \leqslant \max _{0 \leqslant m<3 n}|\hat{h}(m)|\left(\sum_{j=0}^{2 n-1}(1+j)^{2 \alpha}\left(\sum_{m=|n-j|}^{n+j} D(j, m, n)\right)^{2}\right)^{1 / 2} \\
& +c(1+n) \max _{j-n \leqslant m \leqslant n+j} D(j, m, n)\left(\sum_{j=2 n}^{+\infty}\left(\frac{1}{2 n+1} \sum_{m=j-n}^{n+j}(1+m)^{\alpha}|\hat{h}(m)|\right)^{2}\right)^{1 / 2} \\
& \leqslant c(1+n)^{\gamma}\left(\sum_{m=0}^{+\infty}(1+m)^{2 \alpha}|\hat{h}(m)|^{2}\right)^{1 / 2}
\end{aligned}
$$

\section{References}

[1] R. Askey, Orthogonal polynomials and special functions (Regional Conference Series in Applied Mathematics, SIAM, Philadelphia, 1975).

[2] A. I. Bastis, 'Almost everywhere convergence of expansions of the Laplace operator on the sphere', Mat. Zametki 33 (1983), 857-862. 
[3] F. Cazzaniga and E. Giacalone, 'Hardy's inequality for compact symmetric spaces', Boll. Un. Mat. Ital. (6)2-A (1983), 381-388.

[4] R. R. Coifman and G. Weiss, Analyse harmonique non commutative sur certains espaces homogènes (Lecture Notes 242, Springer-Verlag, Berlin, Heidelberg and New York, 1971).

[5] R. R. Coifman and G. Weiss, 'Extension of Hardy spaces and their use in analysis', Bull. Amer. Math. Soc. 83 (1977), 569-645.

[6] L. Colzani, 'Hardy spaces on unit spheres', Boll. Un. Mat. Ital., to appear.

[7] M. Cowling and G. Mauceri, 'On maximal functions', Rend. Sem. Mat. Fis. Milano 49 (1979), $79-88$.

[8] B. Dreseler, 'Norms of zonal spherical functions and Fourier series on compact symmetric spaces', J. Funct. Anal. 44 (1981), 74-86.

[9] R. Gangolli, 'Positive definite kernels on homogeneous spaces and certain stochastic processes related to Levy's Brownian motion of several parameters', Ann. Inst. H. Poincarè Sect. B 3 (1967), 121-225.

[10] S. Helgason, Differential geometry and symmetric spaces (Academic Press, New York and London, 1962).

[11] C. Meaney, 'Divergent Jacobi polynomial series', Proc. Amer. Math. Soc. 87 (1983), 459-462.

[12] C. Meaney, 'Localization of spherical harmonic expansions', Mh. Math. 98 (1984), 65-74.

[13] D. M. Oberlin and E. M. Stein, 'Mapping properties of the Radon transform', Indiana Univ. Math. J. 31 (1982), 641-650.

[14] J. Peyrière and P. Sjölin, 'Regularity of spherical means', Ark. Mat. 16 (1978), 117-126.

[15] P. Sjölin, 'Regularity and integrability of spherical means', Mh. Math .96 (1983), 277-291.

[16] E. M. Stein, 'Maximal functions: spherical means', Proc. Nat. Acad. Sci. U.S.A. 73 (1976), 2174-2175.

[17] G. Szegö, Orthogonal polynomials (3rd edition, Amer. Math. Soc. Colloquium Publ. 23, 1967).

\author{
Dipartimento di Matematica "F. Enriques" \\ Università degli Studi di Milano \\ via C. Saldini, 50 \\ 20133 Milano
}

Italia 